\title{
The Concept of Sustainable Manufacturing Maturity System (SMMS)
}

\author{
${ }^{1}$ Ali-Khusein and ${ }^{2}$ Urquhart \\ ${ }^{1,2}$ First Moscow State University, Russia. \\ 1alikhusein60@gmail.com
}

\begin{abstract}
ArticleInfo
Journal of Machine and Computing (http://anapub.co.ke/journals/jmc/jmc.html)

Doi : https://doi.org/10.53759/7669/jmc202101013

Received 30 December 2020; Revised form 31 January 2021; Accepted 28 March 2021; Available online 05 April 2021. (C)2021 The Authors. Published by AnaPub Publications.

This is an open access article under the CC BY-NC-ND license. (http://creativecommons.org/licenses/by-nc-nd/4.0/)

Abstract - This paper addresses the concept of Sustainable Manufacturing (SM), which represents an innovation in the fields of engineering spanning in the service and product lifecycle. This concept focusses on minimizing negative impacts to the environment, natural resources and energy conservation to ensure consumers, communities and employees are sound, economically. The obligation towards SM is multidimensional. Engineering firms require effective tools that will plan for and assess SM's maturity for achievement of intermediate, long-term and short-term objectives. These maturity models' value will depend on consistent set of test models and performance indicators, which can be quantifiably evaluate with particular uncertainties. This research contribution proposes the Sustainable Manufacturing Maturity System (SMMS), with focus on efforts and concepts required for the development of the system. The proposed scheme is based on Lifecycle Management (LM) measurements, tools and techniques.
\end{abstract}

Keyword - Sustainable Manufacturing (SM), Sustainable Manufacturing Maturity System (SMMS), Lifecycle Management (LM)

\section{INTRODUCTION}

This research contribution focusses on efforts and concepts to propose the Sustainable Manufacturing Maturity System (SMMS). The concept is more compared to sourcing renewable energies and recycling materials as typically perceived; it necessitates lifecycle brainstorming and holistic approaches. Engineering firms in the modern age fall short of amicable and comprehensive frameworks, which incorporate Sustainable Manufacturing (SM) practices for toxic materials, landfills, energy usage and design practices. Moreover, enough support is not provided for quantifiable assessment approaches to identify and evaluate areas of improvement for conformance of standards.

Sustainability is typically acknowledged as the bottom line of profitability in the economy, which also enhances social responsibility [1]. The past technologies are being evaluated as a fourth sustainability dimension. SM is defined differently with reference to the context of research. However, the concept widely implies to the enhancement of innovative manufacturing technologies and sciences, which span the lifecycle of services and products to minimize negative implication of the environment, conserve natural resources and energy to ensure that they are safe for consumers, communities and employees. In the modern age, the move towards SM has become a norm and not a typical practice or initiative. Recently, there has been a trend where international engineering firms are declaring their corporation sustainable development principles. Eco-friendly design, green design or ecological designs have been foundational in various industrial fields, not just engineering.

Design for Ecosystem (DfE), Extended Producer Responsibilities (EPR) and Life Cycle Assessment (LCA) are catching up and novel awareness. The awareness is being formed in the mindsets of consumers and producers. SM has become a strategic approach as engineering firms start to consider applying novel approach to structure efficient application of resources, ensure compliance of standards concerning health and environment, and develop the manufacturing of services and productions. Most of the manufacturing firms are constantly investing in research efforts towards minimization of energy, wasters and resources, remanufacturing and reusing. Engineering firms are really challenged to effectively manage the complete life cycle of products (i.e. Lifecycle Management (LM) [2].

LM defines the traditional focus on sites of production and processes of manufacturing so that the economic, social and environmental impacts of products over its complete life cycle is considered, incorporating the end-of-use and consumption stage. The objective of this is to minimize the product's resource usage and emission to the ecosystem, including improving social economic performance in its life cycle. LM, as shown in Fig 1, allows the designers of products, services providers, individual agents and government agents to make choices for long-term management.

For SM to be achieved, the complete lifecycle of products have to be considered, since engineered products may have completely varied ecological implication during various phases of their life cycle. Over the past few decades, ecological consciourness focus on pollution prevention and the management of wastes, but the approaches only address the minimizaton of possible ecological implications withouth focussing on product designs. Z. Lu in [3] utilizes medical 
metaphor, to convey that the traditional method alleviates symptoms without focussing on the purposes for the diseaase. It is fundamental to incorporate effective measures and tools, which integrate the ecological impacts into the process of product development.

According to J. Hiddink, S. Jennings And M. Kaiser in [4], engineering firms have started to design ecological impacts out of manufacturing processess and products. LM defines the organizational dimesnion of lifecycle methods. In represents integrated concepts for the management of the general lifecycle of services and goods towards sustainable consumption and production. Generally, LM is making product sustainablity and lifecycle thinking operations for engineering firms through progressive enhancements of product processes and systems. It should be considered that LM is not a single methodology or tool, but a management scheme for gathering, disseminating and structuring product-based data from different prograns, tools and concepts incorporating social, economic and ecological aspects of product and their respective lifecycles.

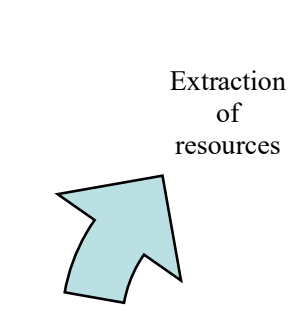

Disposal
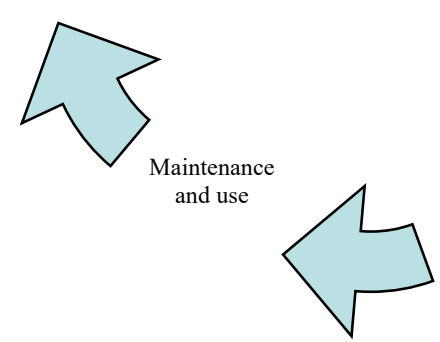
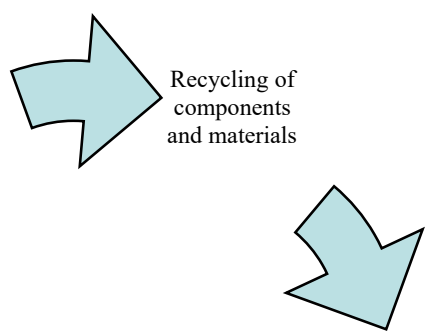

Design and production

Fig 1: Lifecycle management and thinking

LM guide articulates some fundmaental principles and definitions to LM, an explanation of the significance and general scope of intervention, which various areas have in the engineering firms, and a progressive guide, adaptable to companies of any sizes, to support LM integration in the process of management. Different approaches have been followed by engineering firms to apply LM in their respective operations for various applications and to incorporate tripple bottom life aspects into the organizational context. These tools and strategies include Design for Ecosystem (DfE), Lifecycle Costing (LC), Green Procurement (GP), Carbon Footprint Evaluation (CFE), Ecological Performance Indicators and Social Sustainability Approaches. These are in addition to fundamental organizational approaches for actual implementation.

Lifecycle Evaluation represents a procedure to assess the ecological burden associated with activity, process and product through the quantification and identification of materials and energy utilized and wastes released to the ecosystem. LCA producedure also evaluates and identifies the opportunity to impact ecological advancements. The evaluation incorporate the complete lifecycle of products, process or activities, encompassing processing and extracting raw materials; distribution, transportation, manufacturing; maintenance, re-use, use; recycling and complete disposal.

LCA can generate data for production and consumption choices since it evaluates the implications of services and goods. In reference to ISO 14040, LCA incoprorates four stages as shown in Fig 2.

The issue and its boundaries, in the goals and scope stage, are defined. In the inventory phase, materials are utilized, procedures are executed, wastes are produced at every phase of the product's lifecycle. In the assessment phase, LCA values are used to evaluates, weigh and normalize the implications of the products in a single or multiple category. In the last phase, ie interpretation, reviewers interpret assessment results, and draws conclusions before drawing recommendations. Ecological LCA and social LCA are two complementary methods, each of them offering there opinions of the product's life cycle implication. Both are approaches of wide-range sustainability toolbox, which differ with respect to their scope (lifecycle) and the focus of the product.

Lifecyle Costing (LC) generates data on the costs throughout the lifecycle of products. Traditional LC is an approach of evaluating the overal costs of products (services and goods) provided throughout its lifecycle from acquisition to disposal, incorporating designing, installation, operation, maintenance, recycling and the process of disposal. Ecological LC extends ancient $\mathrm{lC}$ in a manner that it evaluates the costs connected to the lifecycle of products, which are covered by a single or 
multiple actors in the lifecycle of products. The actors incorporate the end-users, end-of-life actors, customers, manufacturers, and suppliers.
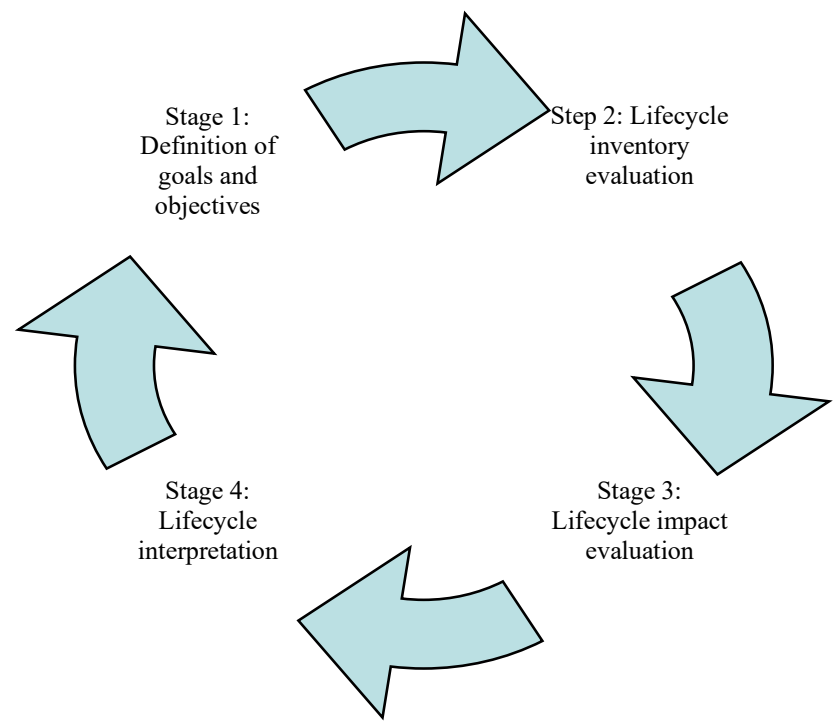

Fig 2: Stages of LCA according to ISO 14040

The remaining section is organized as follows: Section II presents a background analysis of the research. Section III is the literature review section. Section IV presents a critical analysis of the research. Section V present the discussion of research and implementation issues, Lastly, Section VI concludes the paper and provides future directions.

\section{BACKGROUND ANALYSIS}

This research paper proposes a concept of the Sustainable Manufacturing Maturity System (SMMS). This is a concept that is the same or more to sourcing of renewable energy and recycling materials. It requires critical analysis of lifecycles following holistic approaches. Engineering companis today fall short of comprehensive and amicable systems that include SM practices for design practices, energy utility, landfills and toxic materials. Apart from that, support has not been provided enough for the quantifiable evaluation methods meant to identify and assess the segments of developments for standard conformity.

The proposed system is based on the initial model, which has briefly been described in this research. The initial model can help on creating awareness to reduce greenhouse gasses and carbon-dioxide emission. The initial concept is costeffective. This paper follows the initial model to propose a sysrem that is based on the concept of maturity model. The main purpose of the proposed model is to provide high-degree assessment tools for SM efforts. It does not necessitate larger efforts to produce high-degree assessment since it might facilitate its use, mostly by SMEs. This proposed system incorporate particular levels.

The first level is the 'SMMM Level O, which is the initial level. Its default condition for larger numbers of firms is business as usual with limited particular activities for enhancing sustainability incorporate no particular activities for enhancing the efficiency of resources, that is, to minimize the input of materials, water, and energy relative to outputs. Most of the manufacturers have been stimulated by competitive pressure to implement lean manufacturing or enhance the minimization the consumption of energy and costs.

The second level is SMMM Level I i.e. Complaints. This level concentrates on the voluntary compliance of ISO14001-5 standards, which define the implementation of EMS and guarantee of proper application through the process of auditing. Another ISO-14031 assesses the ecological performance of the management scheme. The third level is SMMM Level II i.e. Baseline. This level focusses on the capture of the present performance based on the application of indicators of sustainable manufacturing to act as the baseline for quantifying future developments.

The fourth level is SMMM Level III i.e. improved. This level is capable of identifying manufacturers who have created substantial progress over SM. It necessitates the identification of best performance on the chosen indicators attained by other manufacturers The fifth level is the SMMM Level IV i.e. Best in Class. This level identifies the producers who have attained best in class performances. However, the issue of identifying best-in-class performance is evaluated in the upcoming section. This research paper ends with a discussion of the research and implementation issues that are relevant to the research based on the implementatiion of the proposed model.

\section{LITERATURE REVIEW}

G. Finnveden in [5] evaluates the approach of the European commission, which presented a comprehensive guidance to users regarding the presently-available LCA tools. This was a list of LCA resources, which is given by the Agency of 
environmental protection in USA. Some of these tools that incorporate the LCA approach consider different academic and commercial environmental evaluation tools. Recent reports regarding the sustainability design, the extension of the early ecodesign reports, outline the gradual change of ecodesign to the Design for sustainability concept.

T. Fukushima, K. Takasu And J. Idogawa in [6] also evaluate the design for environment and ecodesign in a comprehensive manner. Product design tools based on LCA based data exist in different forms such as design of sustainability and ecodesign. The researchers widely discuss the design of the ecosystem at an enterprise dimension. W. Schmid in [7] has reportedly included ecodesign approaches alongside LCA and other fundamentals for ecologically conscious designs. A number of point remedy suppliers provide the tools like insight, which incorporate standards: Restrictions of dangerous substances (RoDS), Wastes Electrical and Electronic Equipment (WEEE), End-of-Life Vehicles (EoLV) and Registration, Assessment and Evaluation of Chemicals (RAEC). Software Engineering Body of Knowledge (SEBoK) brings insights into the usage of software engineering tolls for technological segments.

L. Jun in [8] agrees that it is fundamental to embrace a procedural technique to objectively identify, predict and evaluate the enhancements segments for the conformance of standards in the manufacturing segment. R. Serek in [9] proposes the Quality Management Maturity Grids (QMMGs) for the application by an organization or business as the benchmark regarding how mature its procedures are, and the manner in which they have been embedded in their culture based on the product's quality management or service management framework. This was the most traditional inspiration meant to develop the models of maturity.

G. (GCSCC) in [10] confirms that traditional inspiration was meant to enhance maturity models. The Capacity Maturty Models (CMMs) are capable of supporting firms in shifting towards the upcoming level of change in business management. CMMs represents the reference models of mature practices in a particular discipline utilized to evaluate groups' capability to perform that particular discipline. Capacity Maturity Model Integration (CMMI) from Organizational Project Management Maturity Framework (OPMMF) and Software Engineering Institute (SEI) are samples of two maturity frameworks, which address sustainable performance enhancements.

R. Bhatnagar in [11] defines CMMI are a reference framework with the gathering of the best practices from the various disciplines of system and software engineering, incorporated product management and supplier management development. CMMI can be utilized to control the process enancement over the project, a division or a complete organization. It aids in the integration of inter-organizational functions, set procedure improvemenet priorities and goals, provide guidanc for quality procedures, and provide reference points for appraising present processes is standardized for organizations searching to enhance their capacity meant to deliver their approaches through projects. By handling all the organizational projects as an investment in the portfolio and applying best practices meant to manage the initiatives at multiple leveles, an organization can attain their aims and align their resources to operate the things, which matter the most.

$\mathrm{K}$. Rose in [12] evaluates maturity frameworks, incorporating OPMMF and CMMI, give mechanisms for organizations to evaluate themselves over best practices, create development plans and identify gaps meant to cover the gaps with continuos developments. Organizations can attain sustainable developments by embracing OPMMF and CMMI or both. With CMMI's detailed coverage of systems, supplier management, OPMMF wide coverage of projects and software engineering, portfolio and program management, two frameworks provide wealth of knowledge.

J. Viana and C. Mota in [13] argue that every framework comes with evaluation methods that provide various perspectives to reveal potentials and identify needs. CMMI gives a system engineering, supplier management best practices perspective, where OPMMF provides the perspective, which designates best practices of organizations, which attin their strategic aims through successful developments. However, as a group moves from the complaint approach towards sustainabilty, capability, higher maturity level are essential for successful execution. Other than the introduced frameworks, there are PM-CMM, which defines the PM level maturity and capability.

S. Shivankar, G. Kakandikar and V. Nandedkar in [14] projected to quatify situations of PM in a firm and address eight elemenets of PM namely: applications, equipment, techniques, organizational problems, human resources, information and data, processess and products. For every element, the level is evaluated through the examination of multiple parameters indicative of maturity and capability, to quantify firm's PM situation on industry-standards, vendor-independent scales. More recent drafts reported by the department of defense in US discusses the level of manufacturing readiness elaborately to define present dimensions of manufacturing maturity, identify the shortfalls of maturity, associateed rists and costs and provide basis for manufacturing risk management and maturation.

G. Harris, A. Yarbrough, D. Abernathy and C. Peters in [15] have presented a documentation, which provides the best practices for conducting evaluations for manufacturing preparedness. Even though designing certainly for programs of acquisition managers, documentation could possibly prove fundamental towards the identification and conducting manufacturing evaluations in general.

\section{CRITICAL ANALYIS OF SMMM}

Manufacturing firms require dedicated and appropriate tools to significantly plan for and assess sustainable manufacturing maturity lifecycle objectives. The maturity framework tailored exactly through LM techniques and tools such as LCA, manufacturing unit procedures, checklists, performance standards and indicators, can assist interest manufacturity firms to assess their maturity and effectively facilitat benchmarking of manufacturing procedures. The 
projected research idea is the enhancement of dedicated maturity framework to gauge the procedure of sustainability in manufacturing firms.

Manufacturing firms to assess sustainability could utilize SMMM. For SMMM enhancements, we have drawn the inspirations from the initial developed and implemented maturity frameworks. SMMM will take system approaches meant to incorporate inter-organizational elements, set manufacturing procedures, development priorities and goals, issue guidance for quality manufacturing procedures, and provide reference points for appraising sustainability in the present manufacturing procedure.

\section{A. Initial Model}

SMMM can aid in the development of awareness to minimize carbon dioxide (including greenhouse gasses) emission to the economy in a cost effective manner, but also add to the recycling and conservation of energy. In this research, we purpose to present some initial opinions and dialogue sessions regarding the capacity maturity model for sustainable manufacturing and how they can be developed based on LM tools to assess the levels of maturity. We project the process segment, integrated best practices and the evaluation techniques with LM tools such as LCA with more significant manufacturing unit procedures, performance standards and indicators for SMMM. More challenging issue is the identification of the maturity level with comprehensive metrics and indicators. Fig below signifies initial schematic as the first approach towards the enhancement of SMMM.

Manufacturing components and products typically necessitates the incorporation of unit procedures or individual operations such as surface treatment, machining and casting essential for the production of finishes goods through the transformation of raw materials and incorporating the values to work pieces as it is considered as finished product. Unit process and benchmarking templates can be structured to support the standardization of processes and the manufacture of best practices reflecting the resources, processes and products. National Research Council has previously identified five varied unit procedures:

- Mass change procedures that add or remove material by chemical, electric and mechanical means (incorporated are traditional procedures of plating, shearing, grinding and machining, as well as non-traditional procedures as electro-chemical machining, electro-discharge and water-jet)

- Stage-change procedure that produce solid segment from material originally in vapor and liquid phase (normal samples are metal casting, composite manufacturing by injection and infiltration molding of polymers).

- Structure-change procedure that alters the micro-structure of work pieces, either via its localized segment or bulk area such as its stress relief, surface hardening or heat treatment. These are normal processes within this family; family also incorporates stage transitions in the solid conditions e.g. precipitation stiffness.

- Deformation procedure that alters the shape of the solid work pieces without transforming its composition or mass (classic bulk-forming metalwork procedures of forging or rolling grouped as shot-peen sheet-creating procedures e.g. ironing or deep drawing).

- Consolidation procedures that interlink materials like solid sections, filaments and particles to create a solid segment or component (polymer-matrix composite, ceramic molding, powder metallurgy and permanent joining procedures e.g. brazing and welding). Researchers have evaluated the same taxonomies of manufacturing procedure.

\section{B. The Proposed Model}

\section{1) Projected Concept for the Maturity Model}

The obligation of our projected concept for the maturity framework is to provide high-level evaluation tool for the condition of sustainable manufacturing efforts. It does not require large efforts to produce high-level evaluation since it might encourage its usage, certainly by SMEs. It has to provide credible evaluations, which can be contrasted across different companies and manufacturing locations. The desired features stimulated us to project the following criteria in structuring of the concept:

- Incorporate standardized approach for evaluations, which are utilized in the evaluation of maturity

- Incorporate fewer selected indicators for evaluation instead of larger multitudes of indicators.

A structure has been suggested to focus on the compliance and establishment of approaches to the baseline of the status at a lower level to the chosen indicators at a higher level. This is the same to the structured utilized by ANSI-MTS. The ideology of utilizing compliance at the initial levels is also the same to the ideology of prerequisites in UL-880 standard. The identified efforts, ANSI-MTS, and UL-880 standards have a significant scope compared to the projected framework. Moreover, like ANSI-MTS, it is considered that the present standards be used to evaluate the compliance at a lower level instead of creating novel measurement processes. Utility of the present standards will also aid in achieving correspondence with other efforts, which have referred to the same standards. For instance, GRI model has structured have presented documents explaining linkages with ISO standards. 
The projected concepts evaluate maturity with reference to the performance as determine with the chosen indicators instead of the process features. The model, which can support manufacturing with reference to the maturity of the manufacturing procedures, may be challenging to attain because of the varieties over a wider domain within the manufacturing. Certainly, the projected usage of compliance to standards at low maturity levels indirectly evaluates the utility of some procedures. Nonetheless, compliance in this manner is to the measurement procedure for the chosen metrics and not any standardized manufacturing procedures. It might be considered that a firm may attain the projected performance temporarily with ad hoc procedures. This concern is considered via the use of shifting targets at high maturity levels in the projected concept.

A firm cannot attain performance every year using ad hoc procedures and will need to establish mature procedures for consistent performance. The emphasis on performance measures therefore indirectly inspires maturity of operations and manufacturing procedures leading to dependable performance. The projected concept for a sustainable manufacturing maturity framework may be utilized to undertake factory level evaluations to determine the present sustainability over collective defined levels and determine the development chances using the next maturity level as a target. Fig 1 below represents the projected concept of the model. Level 0 represents the baseline level. Level I and Level II evaluate the capacity of the organization to effectively capture the standard indicators for water and energy utility and the efficiency of materials. Level III and Level IV evaluate and determine the factory journey to enhance sustainability through the comparison of indicators with those best in the integrated industry group. Every level has been discussed comprehensively below. The model has to be determined using the indicators within the manufacturing segment through associated industry organization.

\section{a) SMMM Level O (Initial)}

The default condition for larger numbers of firms is business as usual with limited particular activities for enhancing sustainability, incorporating no particular activities for enhancing the efficiency of resources, that is, to minimize the input of materials, water, and energy relative to outputs. Most of the manufacturers have been stimulated by competitive pressure to implement lean manufacturing or enhance the minimization the consumption of energy and costs. Whereas ad hoc efforts may be considered to have quantifiable enhancements, the measurement processes utilized may not be comparable or standard, and therefore, the gathered indicators are challenging compared to other similar manufacturers. There might be limited organizational support or awareness for applying systems that will track indicators for the efficiency of resources.

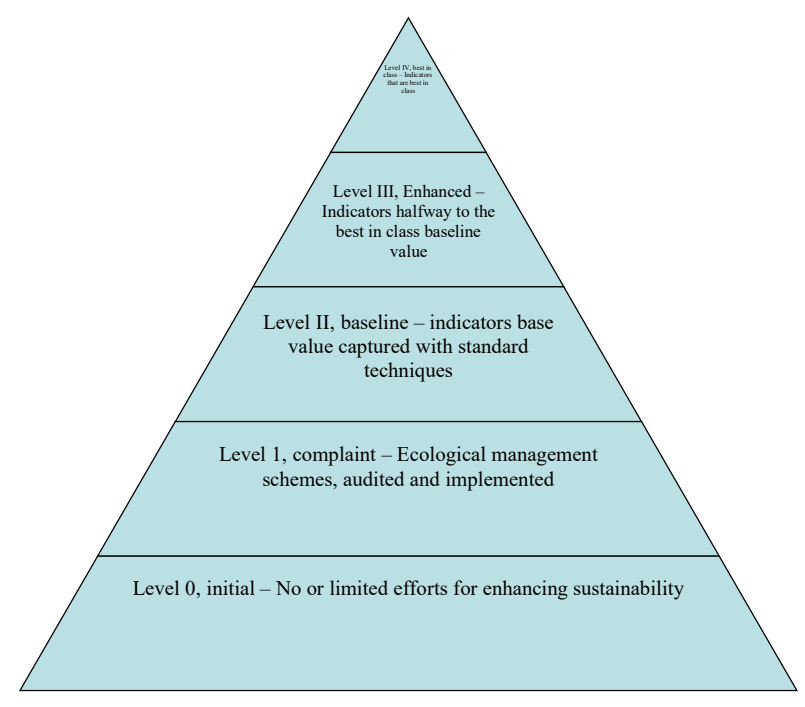

Fig 1: Projected Concept for a Sustainability manufacturing maturity model (SMMM)

\section{b) SMMM Level I (Complaints)}

This level concentrates on the voluntary compliance of ISO-14001-5 standards, which define the implementation of EMS and guarantee of proper application through the process of auditing. Another ISO-14031 assesses the ecological performance of the management scheme. Voluntary compliances with the ISO standard will make sure that producers have management infrastructure for gathering credible information, which can be utilized to produce indicators for evaluating maturity level. EMS application has to be edited based on the guidance from ISO-19011-2002 standards. It is recommended that EMS maintenance and implementation efforts have to be incorporated with efforts such as safety and quality of accepting higher values from initiatives. ISO-14001-2004 issues a table that documents the correspondence available between its elements and the elements of ISO-90001-2008, which defines the schemes of quality management to 
highlight that two schemes can be utilized together. Whereas the application of ISO standard is highly recommended, organizations are capable of electing and identifying standards, which are considered essential to their domains.

\section{c) SMMM Level II (Baseline)}

This level focusses on the capture of the present performance based on the application of indicators of sustainable manufacturing to act as the baseline for quantifying future developments. The measurement has to be made with respect to standard approaches from essential ISO standards (ISO-14051 and ISO-14031). ISO-14031 standard gives the terminology for the performance of SM indicators. ISO-14051 standard provide the protocols for material flow cost accountings to capture for usage of energy, materials and loss in both monetary and physical terms and therefore supports the calculation of indicators defined in this research. While we propose three indicators, a list of standards and indicators as a guide has to be finalized with inputs from organizations, which operate on matters essential to the complete manufacturing segment. Moreover, industry organization has to define particular metrics that correspond to the chosen indicators and augment the standards for use in different domains.

\section{d) SMMM Level III (improved)}

This level is capable of identifying manufacturers who have created substantial progress over SM. It necessitates the identification of best performance on the chosen indicators attained by other manufacturers. Moreover, it necessitates the identification of gaps between the baseline of manufacturers and performance, including the best in class performances, including the determination and implementation of the manner to enhance performance of the chosen measures and affirming that producers have progressed towards attaining best in class performance. The main issue of identifying the best in class performances is evaluated in the following section.

Manufacturers will be considered at level III after attaining audited performance at mid-mark between its baseline performance and best in class performances. It has to be noted that this can be achieved through the designing of the shifting target. The manufacturer who attains level III and therefore attains complacent may be grouped below the level because the best in class performance is projected to continually enhance. The manufacturer may to stimulated to enhance the performance level on the chosen measures to maintain at level III. The initial enhancement in the chosen indicators may be attained through the focus on procedures and policies within the manufacturing facilities. The process and product designs may be handled as fixed constraint.

The major focus on SMMM Level III may be on the procedural operation parameters to develop the chosen indicators. These enhances may incorporate the adjustment of machine settings, and application of lean manufacturing procedures. It is projected that the enhancement that upgrade the producers to level III will amount to cost saving and efficiencies, which can then be used for investments for more sustainability progress.

\section{e) SMMM Level IV (Best in Class)}

This level identifies the producers who have attained best in class performances. However, the issue of identifying bestin-class performance is evaluated in the upcoming section. Best in class may be noted with respect to the indicators' range to attain the highest values over all the indicators for a single manufacturer. Alternate method has to be considered based on the input from the manufacturing firms and other relevant stakeholders. For instance, the composite index interlinking three indicators (or random number of indicators chosen with input from the manufacturing industry) may be utilized based on flexibility given for weightage to be changed by industry organization for particular domains. However, this will progress to be a shifting target at various manufacturers in the identified category will proceed on enhancing and therefore it will not permit any producer who has attained level IV as complacent.

It is projected that producers will have to structure deeper efforts to shift to level IV. These deeper efforts may necessitate the drawing of changes in process and product designs, which lead to the enhancement in the chosen indicators for manufacturing operation. For instance, material transformation for the components may permit the usage of a procedure, which utilizes less energy, water and materials. Producers may also require the data from LCA of products that have been manufactured at the industry to address the issues that have been identified with the design of products and manufacturing. With the projected concept, the manufacturers may utilize the maturity models up to the second level. To shift to level III, manufacturers will have to identify the best in class and the group of the same manufacturers. These groups can be identified through trade associations and consortia. Consortia and trade associations may coordinate sharing and collection of data of indicators in the identified class. The arrangements may be placed to share information anonymously without connecting the names of companies at real indicators therefore encouraging participation. The best in class firms and level III may consider themselves to take credit for their work in attaining maturity and enjoy the advantages from the associated client will.

The motivation behind this research proposal to use the performance of best in class as a target is multi-fold. As mentioned earlier, it stops manufacturers from attaining complacent provided the shifting targets the condition of the best in class indicator. It also permits identifying the target, which are realistic for a particular form of processing. It may not be realistic to manufacturers in a high material-intensive or capital intensive firms e.g. semi-conductor processing to place a target on the indicators attained by the firm involving mechanical assembly operation. The class might be refined by geography within the firm sub-class since the geographical area may have a massive influence on the energy utility certainly because of cooling and heating costs for the manufacturing facilities in firms where the manufacturing procedures are not considered as energy intensive. 


\section{DISCUSSION: RESEARCH AND IMPLEMENTATION ISSUES}

The implementation of SM maturity model will incorporate two iterative phases; the evaluation based on the model and the development efforts to shift towards the next levels. The implementation issue can be considered based on two phases. The concept of SM maturity model has been proposed with respect to the present standards, which make it easier to assess the indicators to permit quick evaluation. There are some research issues in this phase. Evaluation for high level in the projected concept involves the main contrast with the same manufacturers and this could pose issues. It is likely that even the same manufacturers have a varied range of products being processed in their factories, therefore making the work of comparison of facilities challenging.

However, research efforts may be required to define steps to change indicators for fair comparisons between the facilities, which make some the same products. Analytical methods e.g. Information Development Analysis (IDA) may possibly be applied for identification of peers within a large group of the same manufacturer. IDA is non-parametric approach, which use linear programming to potentially identify efficient performers with respect to defined outputs and inputs. Identified effective performances are utilized to defined the frontiers that are utilized to potentially benchmark performances of the producer.

Another research issue is posed by the projected usage of many indicators for comparisons for best in class determination at the fourth level of the concepts. A firm may do better depend on some number of indicators whereas worse on some other indicators. A possible approach to mitigate this issue is to develop weighting methods, which interlink the indicators into one index. Nonetheless, such approaches are criticized that choices of weights reveal high arbitrariness degree. A firm associated or consortium might have to acknowledge on the weights that are acknowledged to everyone. Alternative, IDA might be utilized for automated generation of weights, which are viewed as fair to everyone. There are some research segments, which have to be focused on to assist manufacturers in the second assessment phase, i.e. execution stage to enhance sustainability of operations and enhance the level of maturity. The first phase of implementation of the model with respect on the projected concept will permit manufacturers to assess their levels, and most significantly, to motivate them to enhance their level of maturity for SM.

According to the previous section, upon motivation, manufacturers may be able to use manufacturing practices for initial developments. Comprehensive knowledge of the procedure and product interaction may be needed for more development. The research issues are based on the identification of approaches for developing deep knowledge and utilizing this knowledge for substantial development on the chosen sustainability indicators. Data analytics may be required to comprehend the factors, which impact the chosen indicators and the connection between the indicators and factors to device the means to enhance performance. More analytics that are predictive may be required to test the alternative methods for enhancing the performance level and comprehending their implications on all the fundamental aspects of operations.

Manufacturers would need to ensure that the projected developments on the chosen indicators do not have negative implication on some other performance measures. They would also ensure that the projected performance developments would be achieved. In that regard, the research issue for supporting manufacturers in enhancing sustainability whereas excluding performance measures incorporates performance assurance and data analytics. Main implementation issues for the projected concepts for the model of maturity incorporate the identification of groups of the same manufacturers as benchmarking partners for SM indicators and noticing and documenting performances based on best in class as the main target. The discussions in the past sections recommended noticing the partners in benchmarking through trading associations.

Nonetheless, even within one firm's sub-group, eg one identified by North American Industry grouping system, it can be challenging to identify the manufacturing facilities with the same product range as the other. An alternative form of identifying the same manufacturer is through the Product Category Rule (PCR) that is being developed under auspices of the Global Environmental Product Declaration (GEPD) system. PCR is being developed to categorically support the GEPD definition of the considerable product classes. The main purpose of PCR is to permit the contrast of GEPD in the same group that is the same to our intent of comparing indicators for SM maturity model. Fewer PCRs are present now. In that case, in the near future, manufacturers can focus on developing consortium of the same manufacturers. Organizations may focus on working with the GEPD scheme to effectively develop PCR to access larger groups of manufacturers with the same benchmarks and products to enhance SM. These efforts will also aid in ecological labelling of product since this is a key objective of the GEPD scheme. The projected indicators, i.e. material, water intensity, and energy necessitate the identification of resources in the facility and the correspondent value added. Consumption of research has to be easier to determine the state of the manufacturing facility

Nonetheless, value addition may necessity digging into operational and financial data. Corporations declare value addition in the yearly reports; however, these are dependent on the high-level information for the complete firm. Once the firm identifies that one of the indicators are high, it will necessitate delving into product details and correspondent processes utilized to determine the process-product relationship, which has larger consumption of resources compared to others. Identifying water and energy consumption by process-product relationship can be problematic and might necessitate the installation of novel measurement procedures and equipment. There is a developing body of professional literature and academic experts for evaluating the developing of firms towards SM. 
Most of these literature assumptions are a high level and focus on aspects beyond manufacturing operations. These efforts projected the concept of the model, which concentrated on evaluating the maturity level of SM efforts of manufacturing operations at a particular facility. It is projected that the concept is developed with the inputs from stakeholders to focus on generic SM maturity model with the proposed indicators' level, and standards for application at the lowest level. Particular groups in the industry with domain-specific metrics and standards that correspond to indicators in the generic models can therefore develop the model for usage. The versions of the generic models acknowledged by the relevant stakeholders may be structured for consideration as a standard approved globally.

\section{CONCLUSION}

In this research contribution, SMMM has been proposed and utilized to determine the development of sustainability in manufacturing firms. The concept has been envisioned and utilized by firms to categorically asssed their initial and present conditions of sustainability. Plans of action are dedicated to create awarneess in firms and facilitates the significance of manufacturing maturity models. This contribution focus more on the capacity of a single firm, universities and standard organizations. Next, it facilitates the identification of possible manufactiring stakeholders. This has to be fulfilled through workshop dialogues and manufacturing-based conferences. The efforts illustrated in this contribution has been initiated by group meetings with partners that have reviewed evaluation tools that are suitable for SM. This will potentially set a platform that would identify initial models for te real sustainable model (proposed in this research) and their fundamental elements. Therefore, it will necessitate the identification of critical questions at various levels including systemic incoproration of LM techniques and tools. Nonetheless, practical application of the model might be challenging. Defining the steps that should be followed for the control and development of the processes might be challenging. In that case, future research should focus more on SMMM plan and steps that should be arranged in order for manufacturing firms to follow proactive steps during implementation. This will allow future manufacturing firms to gauge and enhance their present levels and opportunities for progress and manufactiring maturity.

\section{References}

[1]. H. Zhang, J. Calvo-Amodio and K. Haapala, "A conceptual model for assisting sustainable manufacturing through system dynamics", Journal of Manufacturing Systems, vol. 32, no. 4, pp. 543-549, 2013. Available: 10.1016/j.jmsy.2013.05.007.

[2]. A. Karkasinas and A. Rentizelas, "Verification and validation: a novel product lifecycle management-based approach", International Journal of Product Lifecycle Management, vol. 12, no. 2, p. 149, 2019. Available: 10.1504/ijplm.2019.107007.

[3]. Z. Lu, "Traditional Chinese medicine "Qing Yi Tang" alleviates oxygen free radical injury in acute necrotizing pancreatits", World Journal of Gastroenterology, vol. 4, no. 4, p. 357, 1998. Available: 10.3748/wjg.v4.i4.357.

[4]. J. HIDDINK, S. JENNINGS and M. KAISER, "Assessing and predicting the relative ecological impacts of disturbance on habitats with different sensitivities", Journal of Applied Ecology, vol. 44, no. 2, pp. 405-413, 2007. Available: 10.1111/j.1365-2664.2007.01274.x.

[5]. G. Finnveden, "On the limitations of life cycle assessment and environmental systems analysis tools in general", The International Journal of Life Cycle Assessment, vol. 5, no. 4, 2000. Available: 10.1007/bf02979365.

[6]. T. FUKUSHIMA, K. TAKASU and J. IDOGAWA, "Environment-conscious life-cycle design (eco-life-cycle-design) for new external thermal insulation systems.", Journal of Advanced Science, vol. 13, no. 3, pp. 187-190, 2001. Available: 10.2978/jsas.13.187.

[7]. W. Schmidt, "Life Cycle Tools within Ford of Europe's Product Sustainability Index. Case Study Ford S-MAX \& Ford Galaxy (8 pp)", The International Journal of Life Cycle Assessment, vol. 11, no. 5, pp. 315-322, 2006. Available: 10.1065/lca2006.08.267.

[8]. L. Jun, "Simulation and Optimization Scheme of Hull Segment Manufacturing Planning Management", International Journal of Engineering and Applied Sciences (IJEAS), vol. 6, no. 6, 2019. Available: 10.31873/ijeas.6.6.2019.06.

[9]. R. Serek, "Service Quality and Process Maturity Assessment", Journal of Competitiveness, vol. 5, no. 4, pp. 43-56, 2013. Available: 10.7441/joc.2013.04.03.

[10]. G. (GCSCC), "Cybersecurity Capacity Maturity Model for Nations (CMM) Revised Edition", SSRN Electronic Journal, 2016. Available: $10.2139 /$ ssrn.3657116.

[11]. R. Bhatnagar, "Relationship Maturity Model Integration (Rmmi)", Indian Journal of Applied Research, vol. 1, no. 12, pp. 149-151, 2011. Available: 10.15373/2249555x/sep2012/52.

[12]. K. Rose, "Book Review: Organizational Project Management Maturity Model", Project Management Journal, vol. 35, no. 1, pp. 59-59, 2004. Available: 10.1177/875697280403500108.

[13]. J. Viana and C. Mota, "Enhancing Organizational Project Management Maturity: a framework based on the value focused thinking model", Production, vol. 26, no. 2, pp. 313-329, 2015. Available: 10.1590/0103-6513.169913.

[14]. S. Shivankar, G. Kakandikar and V. Nandedkar, "Implementing engineering change management through product life cycle management in automotive field", International Journal of Product Lifecycle Management, vol. 8, no. 2, p. 132, 2015. Available: 10.1504/ijplm.2015.070579.

[15]. G. Harris, A. Yarbrough, D. Abernathy and C. Peters, "Manufacturing Readiness for Digital Manufacturing", Manufacturing Letters, vol. 22, pp. 16-18, 2019. Available: 10.1016/j.mfglet.2019.10.002. 